\title{
Investigation of the Propagation Characteristics of Underwater Shock Waves in Underwater Drilling Blasting
}

\author{
Xin Liu $(\mathbb{D}),{ }^{1}$ Wenbin Gu $\left(\mathbb{D},{ }^{1}\right.$ Jianqing Liu ${ }^{(D)},{ }^{1}$ Zhenxiong Wang $(\mathbb{D})^{2}$ \\ Jinglin $\mathrm{Xu} \mathbb{D}^{\mathrm{D}},{ }^{1}$ and Tao Cao ${ }^{1}$ \\ ${ }^{1}$ The Army Engineering University of PLA, Nanjing 210007, China \\ ${ }^{2}$ Unit No. 96863 of PLA, Luoyang 471003, China \\ Correspondence should be addressed to Wenbin Gu; guwenbin1@aliyun.com
}

Received 19 August 2017; Revised 21 November 2017; Accepted 30 November 2017; Published 15 January 2018

Academic Editor: Matteo Filippi

Copyright (C) 2018 Xin Liu et al. This is an open access article distributed under the Creative Commons Attribution License, which permits unrestricted use, distribution, and reproduction in any medium, provided the original work is properly cited.

During the first-stage project of the main channel of Ningbo-Zhoushan Port's Shipu Harbor, underwater shock waves were monitored. By analyzing a typical measured pressure time history curve, the characteristics of underwater shock waves in an engineering context were obtained. We obtained a traditional exponential attenuation formula for underwater shock waves based on the measured data, simplified the model of underwater drilling blasting based on engineering practice, deduced a revised formula for underwater shock wave peak overpressure on the basis of dimensional analysis, established a linear fitting model, and obtained the undetermined coefficients of the revised formula using a linear regression analysis. In addition, the accuracies of the two formulas used to predict underwater shock wave peak overpressure and the significance order of influence and influence mechanism of factors included in the revised formula on the underwater shock wave peak overpressure were discussed.

\section{Introduction}

Underwater drilling blasting has played an important role in the exploitation and utilization of marine resources, the development of the marine economy, and preparation for military conflicts [1]. In the recent years, under developing economies and quickening paces of construction, the scale of underwater drilling blasting projects has been expanding, bringing significant increases in charge weight per delayed interval, with the results that blasting hazards are being increasingly taken seriously [1-4]. The characteristics of underwater shock waves arising from underwater drilling blasting are related to the weight of charge in blast hole, the relationships between the locations between charges and water bodies as well as the rock mass, hole spacing, borehole diameter, initiation method, and other blasting parameters, with reference to a variety of media that include air, water, rock, and soil. In particular, for underwater drilling blasting in engineering contexts in complex sea areas, underwater shock waves are subjected not only to the influences of interference from the sea surface, seabed, and surrounding environment but also to factors such as the water viscosity, sound velocity (water temperature) distribution, sea surface winds and waves, velocity and direction of tidal flows, tidal range, and specific construction circumstances. Therefore, the study of underwater shock waves is so complex that their theoretical study lags far behind the development of engineering practices $[1,4,5]$.

In combining with the underwater drilling blasting project for the anticollision belts of the Road Bridge over the Yangtze River in Wanzhou, Peng et al. studied the effect of elevation on predictions of blast vibration frequencies using ANSYS/LS-DYNA simulation software [6]. Gu et al. developed a type of water bottom vibration monitor and investigated the propagation and attenuation laws of water bottom vibrations induced by underwater drilling blasting on the basis of actual tests in the field [7]. Tripathy and Shirke studied a specific hard rock dredging case in an Indian port in relation to issues that included controlling blasting hazards in underwater drilling blasting [8]. Sun et al. investigated the characteristics of underwater shock waves by undertaking experiments on underwater drilling blasting 
in shallow undersea [9]. In combining with a pile foundation blasting excavation project at the Shanghai Donghai Bridge, Guo numerically simulated and analyzed rock failure processes in underwater drilling blasting using ANSYS/LSDYNA [10]. Wang numerically simulated the propagation process to obtain propagation laws of underwater shock waves originating from underwater drilling blasting using ANSYS/LS-DYNA and then studied the influence of different detonation methods, stem lengths, and explosive categories on the effects of underwater drilling blasting and shock wave parameters [11]. Chai used numerical simulation software to study the propagation characteristics and attenuation laws of underwater shock waves in underwater drilling blasting under restrictions of single free surface or two free surfaces conditions [12].

Numerical simulations, experimental studies, and combinations of those methods are the main methods used in current underwater drilling blasting studies. However, the numerical simulation method neglects a large number of influencing factors in actual engineering environments, and simulation results are therefore clearly insufficient for providing practical engineering guidance. When applied to underwater drilling blasting, the experimental study method mainly focuses on seismic waves, and research on the generating mechanisms and interaction characteristics of underwater shock waves has scarcely been reported. Existing studies on underwater shock waves in underwater drilling blasting under engineering contexts, especially for studies under complex sea conditions, mostly use underwater acoustic detection systems to measure sound pressures at distance, which greatly limits the results of those studies due to marine environmental noise and restricted sampling frequencies. Moreover, coefficients $K$ and $\alpha$ in the underwater shock wave peak overpressure formula, which represent the relationship between peak overpressure and environment, maximum charge, and distance from the measurement point to the charge, are not universally applicable because they vary in different engineering contexts. Therefore, the study and real-time monitoring of underwater shock waves arising from underwater drilling blasting in blasting engineering are meaningful not only in theory but also in guiding engineering practice to improve energy utilization rates and control blasting hazards $[1,4,13]$.

To study the characteristics of underwater shock waves in underwater drilling blasting under complex sea conditions, a type of effective test method based on actual field test conditions was established, and large numbers of underwater shock waves were measured. Combined with the measured results, mechanisms governing the formation and interaction of underwater shock waves, the attenuation laws governing underwater shock wave peak overpressure, and the regularities of the influences of some blasting parameters on underwater shock wave peak overpressure were analyzed.

\section{Introduction to the Test}

2.1. Overview of the Underwater Drilling Blasting Project. In this project, the rock mass in the reef blasting area is moderately weathered or slightly weathered tuffaceous sandstone,

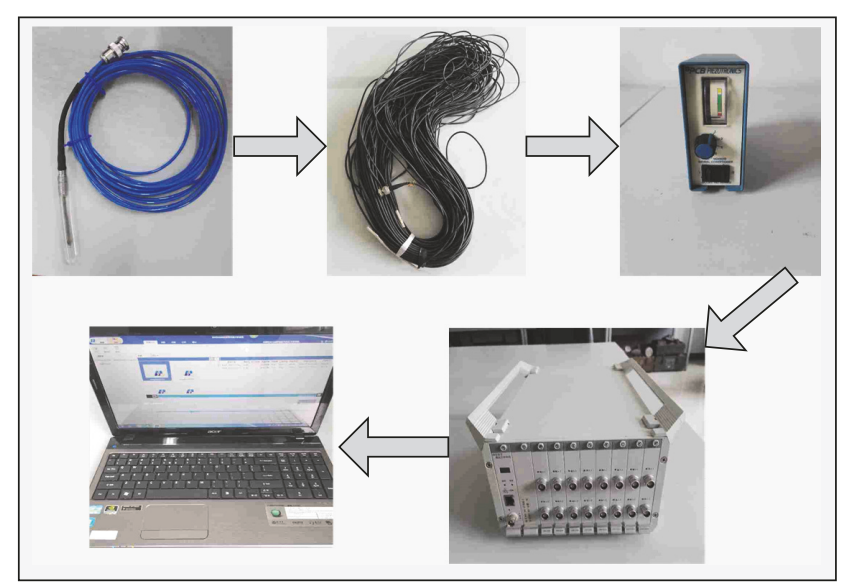

Figure 1: Test system of the shock wave signal.

the tides are regular twice-daily tides, the maximum tidal range is $5.89 \mathrm{~m}$, the average tidal range is $3.23 \mathrm{~m}$, the average high tide is $4.69 \mathrm{~m}$, and the average low tide is $1.48 \mathrm{~m}$. The designed standard altitudes of the bottoms of blast holes were $-10.1 \mathrm{~m}$, the diameters of the blast holes were $115 \mathrm{~mm}$, the depths of blast holes ranged from 3 to $14 \mathrm{~m}$, and the average blast hole extended depths were $2.0 \mathrm{~m}$. The blast holes were drilled vertically, the hole spacings were $2.8 \mathrm{~m}$, the array pitches were $2.2 \mathrm{~m}$, and the designed stem lengths ranged from 1 to $1.5 \mathrm{~m}$, but the actual reserved stem lengths of the blast holes were $1 \mathrm{~m}$ and were not filled during construction. Number 2 rock emulsion explosives were selected as the charges, the diameters of the charges were $90 \mathrm{~mm}$, and the per unit explosive consumption was $1.25 \mathrm{~kg} / \mathrm{m}^{3}$. Two to four detonators were arranged in the bottoms and middles of charges, respectively, in each blast hole, and an initiation mode with interhole and row-to-row short delay was used to detonate one to five rows of blast holes simultaneously.

2.2. Test System. As shown in Figure 1, the underwater shock wave test system was mainly composed of a pressure sensor, low-noise cable, adapter, and dynamic signal acquisition and analysis system. Shock wave signals are collected by the pressure sensor and transmitted to the adapter through a lownoise cable and then through the dynamic signal acquisition and analysis system, which is connected to the adapter, finally reaching the laptop for real-time acquisition and storage. In general, the rise times of underwater shock wave signals are at the microsecond level, and the durations are less than 2-3 ms, which means that there were large demands on the dynamic response characteristics of the test system. Underwater shock wave signals are a type of nonperiodic pulse signals that have very wide continuous spectra and therefore required a wide frequency response range for the test system. Considering the above-mentioned requirements, a $\mathrm{PCB}$ production 138 series A02 underwater free field shock wave sensor was employed in the test. This type of sensor uses a tourmaline crystal as the sensitive element and is equipped with a built-in ICP circuit, which causes it to demonstrate many advantages such as short rise time, high amplitude linearity, smooth waveform, lack 
TABLE 1: Basic sensor performance indicators.

\begin{tabular}{|c|c|c|c|}
\hline $\begin{array}{l}\text { Measurement } \\
\text { range }\end{array}$ & $6895 \mathrm{kPa}$ & Useful over range & $13790 \mathrm{kPa}$ \\
\hline Sensitivity & $0.73 \mathrm{mv} / \mathrm{kPa}$ & $\begin{array}{l}\text { Maximum } \\
\text { pressure }\end{array}$ & $344750 \mathrm{kPa}$ \\
\hline Resolution & $0.14 \mathrm{kPa}$ & $\begin{array}{l}\text { Resonant } \\
\text { frequency }\end{array}$ & $\geq 1000 \mathrm{kHz}$ \\
\hline Rise time & $\leq 1.5$ us & $\begin{array}{l}\text { Low frequency } \\
\text { response }\end{array}$ & $2.5 \mathrm{~Hz}$ \\
\hline Nonlinearity & $\leq 2.0 \% \mathrm{FS}$ & & \\
\hline
\end{tabular}

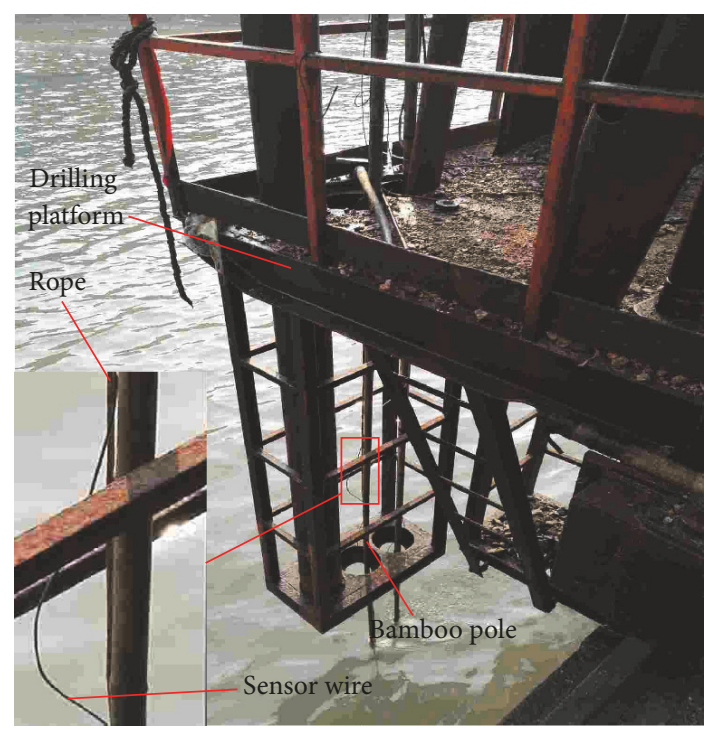

Figure 2: Setup of the sensor.

of resonance, high voltage output, capability for driving long cables, and low noise. The performance parameters of the sensor are shown in Table 1. A PCB production 482 series adapter was used. This type of adapter can provide a stable excitation current to a sensor and decouple DC bias voltage in the AC test signal. A Jiangsu Donghua test corporation production type DH5960 dynamic signal acquisition and analysis system was selected, the bundled analysis software of which supported a variety of functions such as real-time measurement, playback analysis, and data conversion.

2.3. Experimental Setup. Considering the complex sea conditions in Ningbo-Zhoushan Port's Shipu Harbor, our test was carried out on a reef-explosion vessel. As shown in Figure 2, to avoid being pulled, the sensor wire and low-noise cable were loosely fixed on a rope, a certain length of which was fixed on a straight bamboo pole. A pressure sensor was suspended vertically from one end of the bamboo pole and was attached to a small weight (approximately $1 \mathrm{~kg}$ ) to make sure that the rope straightened. The straight bamboo pole was dipped into the water from the drilling platform of the reef-explosion vessel, where the sleeve was placed. The entry depth of the sensor in the water was adjusted by adjusting the entry depth of the bamboo pole. When collecting signals,

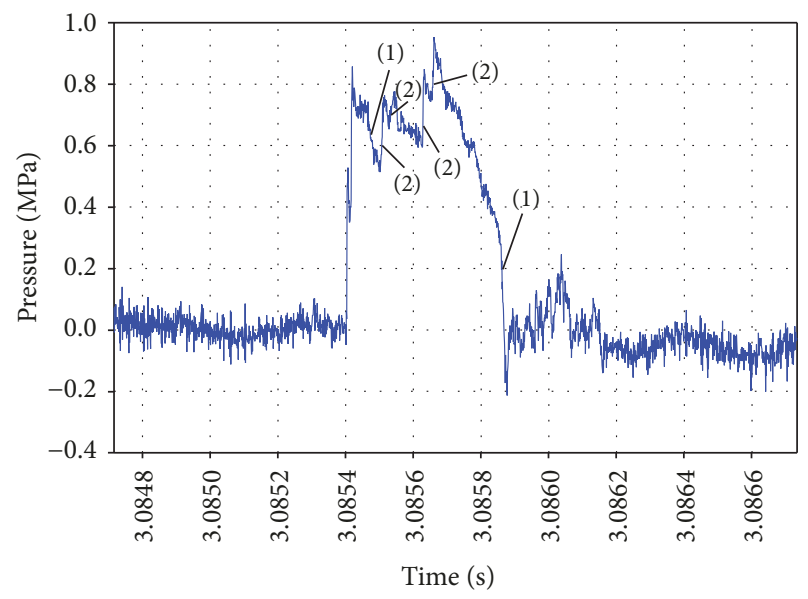

(1) Truncation effect

(2) The mutual superposition of shock wave

FIGURE 3: Typical pressure time history curve of underwater shock wave.

to avoid false triggering in the dynamic signal acquisition and analysis system caused by noise and failure to trigger or missing data caused by too high trigger thresholds, the external trigger mode was chosen. In addition, to compensate for synchronization errors between the signal triggers and charge detonations, the delay setting was set to the negative delay mode. The length of the negative delay was $1 \mathrm{M}$, the sampling length was $8 \mathrm{M}$, and the sampling rate was $1 \mathrm{M} / \mathrm{s}$.

\section{Test Results}

3.1. Pressure Time History Curve of an Underwater Shock Wave. Figure 3 shows a typical measured pressure time history curve of an underwater shock wave. The underwater shock wave shown in Figure 3 was generated by $327 \mathrm{~kg}$ of explosives that were distributed in 14 blast holes with the same delay time setups. Underwater shock waves in underwater drilling blasting mainly consist of two parts: one is the shock waves generated by high temperatures and high pressure detonation products when the detonation products rush out from the upper part of the blast hole to the water and the other is the shock waves generated by stress waves in rock, which are produced when stress waves are refracted into the water and are known as earth-water shock waves [13]. Because the propagation speeds of stress waves in rock exceeded those of the shock waves in water, the earthwater shock waves generally reached the measurement point sooner, which was not shown in Figure 3. The obstacle effect of blasting reef slags heaped on the seabed between the measurement point and explosive source was the primary reason why the underwater shock wave shown in Figure 3 did not contain an earth-water shock wave component. As shown in Figure 3, the underwater shock wave had successive multiple peak values; the shock wave that arrived later was superimposed on the rise or decay stages of the shock wave that had arrived earlier, meaning that the underwater shock waves generated by each blast hole, which had the same delay 


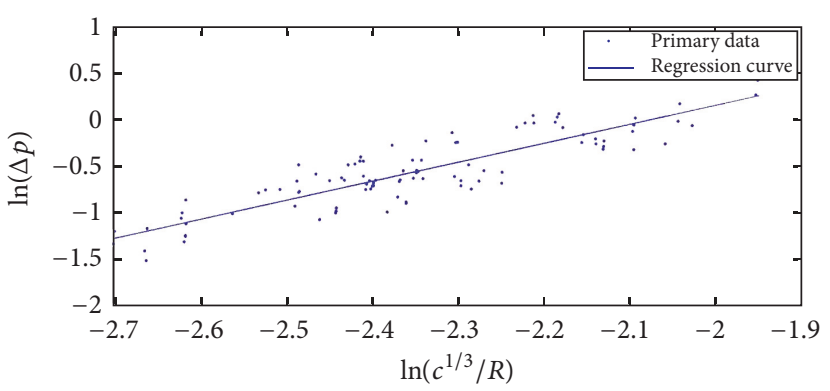

(a)

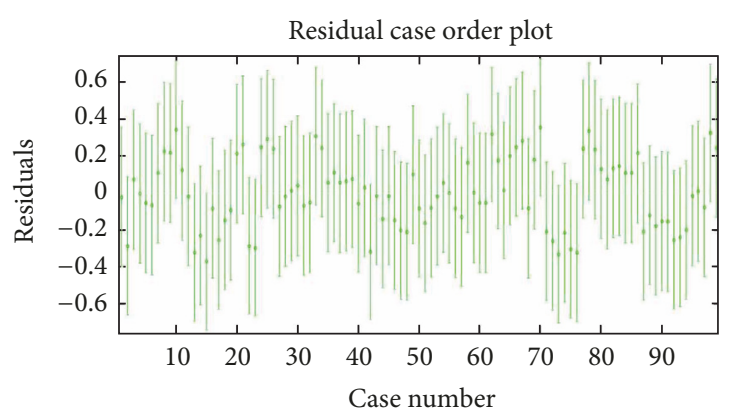

(b)

FIGURE 4: The final regression curve and residuals case order of the shock wave peak overpressure.

times, did not reach the measurement points at the same time. Because of the different lengths of the Nonel tubes connected to each blast hole in the detonation network, the delay time error of the millisecond detonator, and the different propagation distances, the underwater shock waves reached the measurement points successively and were superimposed on each other, and the maximum value of shock wave peak overpressure depended on the superposition of the shock waves to some extent. As shown in Figure 3, the action time of the underwater shock wave positive pressure was much more prolonged than that of the typical underwater shock wave generated by a single blast hole, the decay stage of the underwater shock wave was closer to linear attenuation, and the tail of the underwater shock wave suffered from a reflected sparse wave from the water surface, which caused the "cutoff" phenomenon to occur.

\subsection{The Underwater Shock Wave Peak Overpressure}

3.2.1. Classical Formula and Error Analysis. Assume that the hydrostatic pressure and the initial density of water at the measurement point are $p_{0}$ and $\rho_{0}$, respectively, when the charge is exploded. For the underwater shock wave peak overpressure $\Delta p$ at the measurement point, according to the traditional exponential attenuation formula, assume that

$$
\Delta p=k\left(\frac{\sqrt[3]{c}}{R}\right)^{\alpha}
$$

where $c$ is the total mass of charges with the same delay time. By taking the logarithms of both sides of the equation, we obtain

$$
\ln \Delta p=\alpha \ln \left(\frac{\sqrt[3]{c}}{R}\right)+\ln k
$$

Let $y=\ln \Delta p, x=\ln (\sqrt[3]{c} / R)$, and $b=\ln k$, and then

$$
y=\alpha x+b .
$$

The fit results of the measured data shown in Table 2, which were produced using the MATLAB unitary linear regression analysis tool, are shown in Figure 4. Corresponding to the fit results, the coefficients $\alpha=2.04975$ and $b=$ 4.25993, the correlation coefficient $\gamma=0.8885$, complex

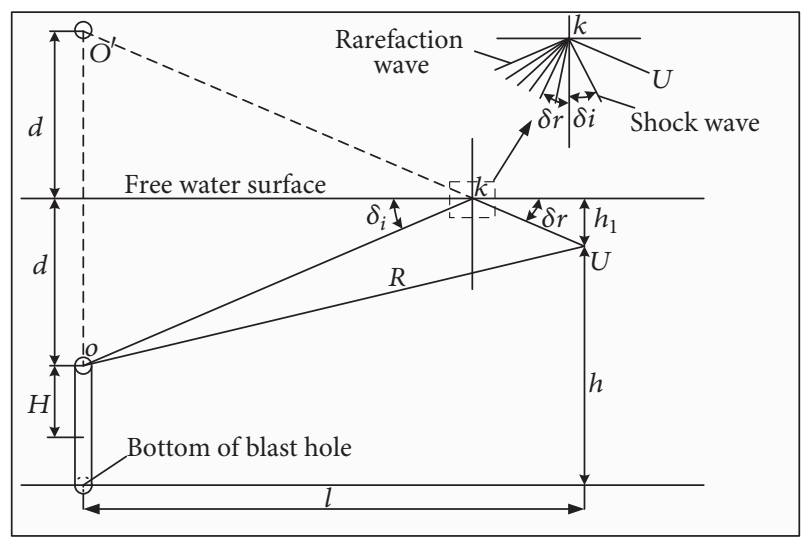

FIgURE 5: Simplified model of underwater drilling blasting.

correlation coefficient $R^{2}=0.7894$, and the estimated standard error $S_{y}=0.1934$. Therefore,

$$
\begin{aligned}
y & =2.04975 x+4.25993 \\
\Delta p & =70.80503 *\left(\frac{\sqrt[3]{c}}{R}\right)^{2.04975}(\mathrm{Mpa}),
\end{aligned}
$$

where $0.067 \leq \sqrt[3]{c} / R \leq 0.142$.

The underwater shock wave peak overpressures were calculated using formula (5), and the calculated values and measured data were compared to obtain the relative error between them. Finally, we found that the relative errors were between $0.07 \%$ and $32.3 \%$, and $50 \%$ of the measurement points had relative errors exceeding $15 \%$. It was therefore obvious that there were very large errors when predicting the underwater shock wave peak overpressures using the fitted exponential attenuation formula; that is, the fitted exponential attenuation formula was unable to adequately describe the attenuation characteristics of the underwater shock wave peak overpressure.

3.2.2. Dimensional Analysis and Revised Formula. To gain a better understanding of the characteristics of the underwater shock wave shown in Figure 5, which was generated from a single blast in underwater drilling blasting, the explosive 
TABLE 2: Test results.

\begin{tabular}{|c|c|c|c|c|c|c|c|c|c|}
\hline$d / \mathrm{m}$ & $H / \mathrm{m}$ & $h_{1} / \mathrm{m}$ & $h / \mathrm{m}$ & $l / \mathrm{m}$ & $R / \mathrm{m}$ & $c / \mathrm{kg}$ & $\delta 1 / \mathrm{rad}$ & $\delta^{*} / \mathrm{rad}$ & $\Delta p / \mathrm{MPa}$ \\
\hline 12.417 & 2.917 & 3 & 14.25 & 60 & 60.73 & 168 & 0.2515 & 0.0377 & 0.506891 \\
\hline 12.417 & 2.917 & 3 & 14.25 & 64 & 64.69 & 168 & 0.2364 & 0.0355 & 0.342659 \\
\hline 9.8 & 4.225 & 3 & 14.25 & 64 & 64.36 & 193 & 0.1974 & 0.0395 & 0.54561 \\
\hline 9.8 & 4.225 & 3 & 14.25 & 60 & 60.38 & 193 & 0.2102 & 0.042 & 0.575542 \\
\hline 8.025 & 5.113 & 3 & 14.25 & 60 & 60.21 & 228 & 0.1817 & 0.047 & 0.61741 \\
\hline 8.025 & 5.113 & 3 & 14.25 & 64 & 64.2 & 228 & 0.1706 & 0.0441 & 0.535263 \\
\hline 6.91 & 3.815 & 1.5 & 12.04 & 61.35 & 61.59 & 207 & 0.1362 & 0.0397 & 0.64834 \\
\hline 6.91 & 3.815 & 2 & 11.54 & 66.35 & 66.53 & 207 & 0.1618 & 0.04 & 0.622727 \\
\hline 8.515 & 3.013 & 1.5 & 12.04 & 61.35 & 61.75 & 237 & 0.1335 & 0.0389 & 0.789 \\
\hline 8.515 & 3.013 & 2 & 11.54 & 66.35 & 66.67 & 237 & 0.1572 & 0.0388 & 0.76387 \\
\hline 6.7775 & 3.881 & 2 & 11.54 & 66.35 & 66.52 & 339 & 0.1315 & 0.0461 & 1.077255 \\
\hline 6.7775 & 3.881 & 1.5 & 12.04 & 61.35 & 61.58 & 339 & 0.1341 & 0.047 & 0.921399 \\
\hline 8.865 & 2.838 & 2 & 11.54 & 66.35 & 66.7 & 108 & 0.1623 & 0.0295 & 0.177756 \\
\hline 8.865 & 2.838 & 1.5 & 12.04 & 61.35 & 61.79 & 108 & 0.1674 & 0.0305 & 0.364038 \\
\hline 9.85 & 3.975 & 0.4 & 16.4 & 54 & 54.82 & 174 & 0.1876 & 0.036 & 0.47628 \\
\hline 9.85 & 3.975 & 0.4 & 16.4 & 59 & 59.75 & 174 & 0.172 & 0.033 & 0.437284 \\
\hline 10.075 & 3.863 & 0.4 & 16.4 & 54 & 54.86 & 168 & 0.1916 & 0.0355 & 0.393435 \\
\hline 10.075 & 3.863 & 0.4 & 16.4 & 59 & 59.79 & 168 & 0.1757 & 0.0326 & 0.370492 \\
\hline 12.323 & 2.314 & 3.4 & 12.55 & 61 & 61.65 & 174 & 0.2523 & 0.0386 & 0.475177 \\
\hline 12.323 & 2.314 & 0.3 & 15.65 & 53 & 54.35 & 174 & 0.2338 & 0.0358 & 0.517805 \\
\hline 12.778 & 2.086 & 0.3 & 15.65 & 51 & 52.5 & 144 & 0.251 & 0.0347 & 0.544111 \\
\hline 12.778 & 2.086 & 0.3 & 15.65 & 53 & 54.45 & 144 & 0.2419 & 0.0335 & 0.533348 \\
\hline 12.778 & 2.086 & 3.4 & 12.55 & 61 & 61.72 & 144 & 0.2592 & 0.0358 & 0.559859 \\
\hline 11.8 & 2.575 & 3.4 & 12.55 & 61 & 61.58 & 297 & 0.2442 & 0.0467 & 0.967485 \\
\hline 11.8 & 2.575 & 0.3 & 15.65 & 53 & 54.23 & 297 & 0.2245 & 0.043 & 0.726301 \\
\hline 11.8 & 2.575 & 0.3 & 15.65 & 51 & 52.28 & 297 & 0.2329 & 0.0446 & 0.775246 \\
\hline 11.02 & 2.965 & 1.7 & 14.3 & 57.88 & 58.64 & 144 & 0.2155 & 0.0347 & 0.642444 \\
\hline 11.02 & 2.965 & 2 & 13.95 & 57.88 & 58.58 & 144 & 0.2212 & 0.0356 & 0.673955 \\
\hline 11.02 & 2.965 & 3.5 & 12.45 & 57.88 & 58.37 & 144 & 0.2458 & 0.0395 & 0.644505 \\
\hline 10.94 & 3.005 & 1.7 & 14.3 & 57.88 & 58.62 & 147 & 0.2142 & 0.0349 & 0.474167 \\
\hline 10.94 & 3.005 & 2 & 13.95 & 57.88 & 58.57 & 147 & 0.2199 & 0.0359 & 0.501053 \\
\hline 10.94 & 3.005 & 3.5 & 12.45 & 57.88 & 58.36 & 147 & 0.2445 & 0.0398 & 0.520017 \\
\hline 10.68 & 3.135 & 3.5 & 12.45 & 57.88 & 58.33 & 165 & 0.2402 & 0.0417 & 0.579999 \\
\hline 10.68 & 3.135 & 1.7 & 14.3 & 57.88 & 58.58 & 165 & 0.2099 & 0.0365 & 0.51563 \\
\hline 10.68 & 3.135 & 2 & 13.95 & 57.88 & 58.53 & 165 & 0.2157 & 0.0375 & 0.526159 \\
\hline 10.02 & 3.465 & 3.5 & 12.45 & 57.88 & 58.25 & 180 & 0.2295 & 0.0438 & 0.891855 \\
\hline 10.02 & 3.465 & 1.7 & 14.3 & 57.88 & 58.49 & 180 & 0.1989 & 0.038 & 0.799492 \\
\hline 10.02 & 3.465 & 2 & 13.95 & 57.88 & 58.44 & 180 & 0.2047 & 0.0391 & 0.864397 \\
\hline 12.2 & 2.375 & 3.5 & 12.45 & 57.88 & 58.53 & 78 & 0.2649 & 0.0312 & 0.422521 \\
\hline 12.2 & 2.375 & 1.7 & 14.3 & 57.88 & 58.84 & 78 & 0.2349 & 0.0277 & 0.347228 \\
\hline 12.2 & 2.375 & 2 & 13.95 & 57.88 & 58.78 & 78 & 0.2406 & 0.0284 & 0.367169 \\
\hline 11.7 & 2.625 & 1.7 & 14.3 & 57.88 & 58.75 & 117 & 0.2267 & 0.032 & 0.458776 \\
\hline 11.7 & 2.625 & 2 & 13.95 & 57.88 & 58.69 & 117 & 0.2324 & 0.0328 & 0.463055 \\
\hline 11.7 & 2.625 & 3.5 & 12.45 & 57.88 & 58.46 & 117 & 0.2568 & 0.0362 & 0.818814 \\
\hline 7.74 & 3.6 & 3 & 10.94 & 62.6 & 62.78 & 75 & 0.1699 & 0.0314 & 0.301618 \\
\hline 7.74 & 3.6 & 2 & 11.94 & 62.6 & 62.87 & 75 & 0.1543 & 0.0285 & 0.262945 \\
\hline 7.74 & 3.6 & 1 & 12.94 & 62.6 & 62.96 & 75 & 0.1387 & 0.0256 & 0.164646 \\
\hline 7.34 & 3.8 & 3 & 10.94 & 62.6 & 62.75 & 84 & 0.1637 & 0.0331 & 0.311197 \\
\hline 7.34 & 3.8 & 2 & 11.94 & 62.6 & 62.83 & 84 & 0.1481 & 0.03 & 0.219733 \\
\hline 6.39 & 4.275 & 3 & 10.94 & 62.6 & 62.69 & 96 & 0.1489 & 0.0362 & 0.326962 \\
\hline 6.39 & 4.275 & 2 & 11.94 & 62.6 & 62.76 & 96 & 0.1332 & 0.0324 & 0.28819 \\
\hline
\end{tabular}


TABLe 2: Continued.

\begin{tabular}{|c|c|c|c|c|c|c|c|c|c|}
\hline$d / \mathrm{m}$ & $H / \mathrm{m}$ & $h_{1} / \mathrm{m}$ & $h / \mathrm{m}$ & $l / \mathrm{m}$ & $R / \mathrm{m}$ & $c / \mathrm{kg}$ & $\delta 1 / \mathrm{rad}$ & $\delta^{*} / \mathrm{rad}$ & $\Delta p / \mathrm{MPa}$ \\
\hline 6.39 & 4.275 & 3 & 10.94 & 62.6 & 62.69 & 96 & 0.1489 & 0.0362 & 0.326786 \\
\hline 6.39 & 4.275 & 2 & 11.94 & 62.6 & 62.76 & 96 & 0.1332 & 0.0324 & 0.28562 \\
\hline 6.39 & 4.275 & 1 & 12.94 & 62.6 & 62.83 & 96 & 0.1175 & 0.0286 & 0.269675 \\
\hline 7.34 & 3.8 & 1 & 12.94 & 62.6 & 62.92 & 84 & 0.1324 & 0.0268 & 0.243959 \\
\hline 10.575 & 2.588 & 1.8 & 12.95 & 54.41 & 55.11 & 366 & 0.2237 & 0.0513 & 1.19328 \\
\hline 10.575 & 2.588 & 1.1 & 13.65 & 54.41 & 55.22 & 366 & 0.2114 & 0.0485 & 0.987916 \\
\hline 10.575 & 2.588 & 0.7 & 14.1 & 53.41 & 54.32 & 366 & 0.2072 & 0.0476 & 0.945228 \\
\hline 11.106 & 2.322 & 0.7 & 14.1 & 54.41 & 55.4 & 315 & 0.2128 & 0.0442 & 0.887658 \\
\hline 11.106 & 2.322 & 1.1 & 13.65 & 54.41 & 55.32 & 315 & 0.2207 & 0.0458 & 0.949607 \\
\hline 11.106 & 2.322 & 1.8 & 12.95 & 54.41 & 55.2 & 315 & 0.2329 & 0.0483 & 1.024876 \\
\hline 10.445 & 2.653 & 1.8 & 12.95 & 54.41 & 55.09 & 261 & 0.2214 & 0.0459 & 0.855371 \\
\hline 10.445 & 2.653 & 1.1 & 13.65 & 54.41 & 55.2 & 261 & 0.2091 & 0.0434 & 0.785504 \\
\hline 10.445 & 2.653 & 0.7 & 14.1 & 53.41 & 54.3 & 261 & 0.2048 & 0.0425 & 0.775391 \\
\hline 11.817 & 1.967 & 1.8 & 12.95 & 54.41 & 55.32 & 87 & 0.2453 & 0.031 & 0.471585 \\
\hline 11.817 & 1.967 & 1.1 & 13.65 & 54.41 & 55.45 & 87 & 0.2331 & 0.0295 & 0.256385 \\
\hline 11.817 & 1.967 & 0.7 & 14.1 & 53.41 & 54.56 & 87 & 0.2293 & 0.029 & 0.264238 \\
\hline 10.28 & 2.735 & 1.8 & 12.95 & 54.41 & 55.06 & 126 & 0.2185 & 0.036 & 0.522689 \\
\hline 10.28 & 2.735 & 1.1 & 13.65 & 54.41 & 55.17 & 126 & 0.2062 & 0.034 & 0.493013 \\
\hline 10.28 & 2.735 & 0.7 & 14.1 & 54.41 & 55.25 & 126 & 0.1983 & 0.0327 & 0.490539 \\
\hline 8.01 & 3.16 & 3 & 10.33 & 58 & 58.22 & 569 & 0.1876 & 0.0662 & 1.790546 \\
\hline 8.01 & 3.16 & 2 & 11.33 & 58 & 58.31 & 569 & 0.1709 & 0.0603 & 1.547709 \\
\hline 8.01 & 3.16 & 1 & 12.33 & 58 & 58.42 & 569 & 0.1541 & 0.0544 & 1.311747 \\
\hline 9.497 & 2.417 & 1 & 12.33 & 58 & 58.62 & 285 & 0.179 & 0.0421 & 0.979389 \\
\hline 9.497 & 2.417 & 2 & 11.33 & 58 & 58.48 & 285 & 0.1957 & 0.046 & 1.031219 \\
\hline 9.497 & 2.417 & 3 & 10.33 & 58 & 58.36 & 285 & 0.2122 & 0.0499 & 1.073093 \\
\hline 8.455 & 2.938 & 1 & 12.33 & 58 & 58.48 & 114 & 0.1616 & 0.0314 & 0.395802 \\
\hline 8.455 & 2.938 & 2 & 11.33 & 58 & 58.36 & 114 & 0.1783 & 0.0347 & 0.517322 \\
\hline 8.455 & 2.938 & 3 & 10.33 & 58 & 58.26 & 114 & 0.195 & 0.0379 & 0.618029 \\
\hline 4.933 & 5.559 & 0.8 & 14.25 & 70 & 70.12 & 351 & 0.0817 & 0.0398 & 0.520063 \\
\hline 4.933 & 5.559 & 0.5 & 14.55 & 70 & 70.14 & 351 & 0.0775 & 0.0377 & 0.493361 \\
\hline 4.93 & 5.56 & 0.5 & 14.55 & 70 & 70.14 & 405 & 0.0774 & 0.0396 & 0.506443 \\
\hline 4.93 & 5.56 & 0.8 & 14.25 & 70 & 70.12 & 405 & 0.0817 & 0.0418 & 0.568769 \\
\hline 6.22 & 4.915 & 0.8 & 14.25 & 70 & 70.21 & 291 & 0.1 & 0.0362 & 0.413992 \\
\hline 6.22 & 4.915 & 0.5 & 14.55 & 70 & 70.23 & 291 & 0.0957 & 0.0347 & 0.407112 \\
\hline 10.286 & 2.682 & 1.5 & 13.15 & 63.59 & 64.19 & 327 & 0.1833 & 0.0417 & 0.927974 \\
\hline 10.286 & 2.682 & 1.5 & 13.15 & 68.64 & 69.2 & 327 & 0.17 & 0.0387 & 0.873904 \\
\hline 10.286 & 2.682 & 1.5 & 13.15 & 70.41 & 70.95 & 327 & 0.1659 & 0.0377 & 1.056433 \\
\hline 10.813 & 2.419 & 1.5 & 13.15 & 68.64 & 69.27 & 228 & 0.1775 & 0.034 & 0.618254 \\
\hline 10.813 & 2.419 & 1.5 & 13.15 & 63.59 & 64.27 & 228 & 0.1913 & 0.0366 & 0.649844 \\
\hline 10.956 & 2.347 & 1.5 & 13.15 & 63.59 & 64.29 & 180 & 0.1934 & 0.0337 & 0.521943 \\
\hline 10.956 & 2.347 & 1.5 & 13.15 & 68.64 & 69.29 & 180 & 0.1795 & 0.0313 & 0.47424 \\
\hline 10.956 & 2.347 & 1.5 & 13.15 & 70.41 & 71.04 & 180 & 0.1751 & 0.0306 & 0.456139 \\
\hline 8.8375 & 3.106 & 1.5 & 12.55 & 72.5 & 72.87 & 249 & 0.1416 & 0.0342 & 0.521214 \\
\hline 8.8375 & 3.106 & 1.5 & 12.55 & 71.5 & 71.88 & 249 & 0.1436 & 0.0347 & 0.536574 \\
\hline 8.8375 & 3.106 & 1.5 & 12.55 & 62.5 & 62.93 & 249 & 0.1639 & 0.0396 & 0.783273 \\
\hline 8.48 & 3.285 & 0.3 & 13.75 & 58 & 58.57 & 336 & 0.1502 & 0.0419 & 0.729505 \\
\hline 8.48 & 3.285 & 0.7 & 13.35 & 58 & 58.52 & 336 & 0.157 & 0.0438 & 0.797511 \\
\hline 8.48 & 3.285 & 0.5 & 13.55 & 58 & 58.55 & 336 & 0.1536 & 0.0428 & 0.752291 \\
\hline 6.72 & 4.165 & 0.3 & 13.75 & 58 & 58.35 & 219 & 0.1204 & 0.0367 & 0.579942 \\
\hline 6.72 & 4.165 & 0.5 & 13.55 & 58 & 58.33 & 219 & 0.1238 & 0.0378 & 0.578682 \\
\hline 8.53 & 3.275 & 0.3 & 13.78 & 58 & 58.58 & 132 & 0.1511 & 0.0306 & 0.36792 \\
\hline
\end{tabular}


TABLE 2: Continued.

\begin{tabular}{lccccccccc}
\hline$d / \mathrm{m}$ & $H / \mathrm{m}$ & $h_{1} / \mathrm{m}$ & $h / \mathrm{m}$ & $l / \mathrm{m}$ & $R / \mathrm{m}$ & $c / \mathrm{kg}$ & $\delta 1 / \mathrm{rad}$ & $\delta^{*} / \mathrm{rad}$ & $\Delta p / \mathrm{MPa}$ \\
\hline 8.53 & 3.275 & 0.5 & 13.58 & 58 & 58.55 & 132 & 0.1544 & 0.0313 & 0.373628 \\
8.53 & 3.275 & 0.7 & 13.38 & 58 & 58.53 & 132 & 0.1578 & 0.0319 & 0.388874 \\
12.51 & 2.375 & 0.3 & 15.96 & 58.53 & 59.79 & 186 & 0.2155 & 0.0332 \\
12.51 & 2.375 & 0.5 & 15.76 & 58.53 & 59.75 & 186 & 0.2187 & 0.0337 & 0.581431 \\
11.58 & 2.84 & 0.3 & 15.96 & 58.53 & 59.6 & 345 & 0.2003 & 0.0411 & 0.816798 \\
12.22 & 2.52 & 0.3 & 15.96 & 58.53 & 59.73 & 279 & 0.2107 & 0.0382 \\
12.22 & 2.52 & 0.5 & 15.76 & 58.53 & 59.69 & 279 & 0.214 & 0.0387 & 0.970671 \\
\hline
\end{tabular}

source was simplified as a single blast hole located in the center of the blasting area, the average depths of all blast holes with the same delay times were taken as the depths of the blast holes, the average charge length of all the blast holes with the same delay times was taken as the charge length of the blast holes, the average water depth $d$ above all the blast holes with the same delay times was taken as the water depth in the blasting area, and the sum $c$ of the charge weights of all the blast holes with the same delay times was taken as the charge weight of the single simplified blast hole. Point $o$ is the center of the upper end surface of the blast hole, point $o^{\prime}$ is the virtual wave source for the mirror of the free water surface, and point $U$ is the measurement point. In the simplified model shown in Figure 5, $H$ is the distance between the center of the charge and the upper end surface of the blast hole, $l$ is the horizontal distance between the measurement point and point $o, h_{1}$ is the distance between the measurement point and the water surface, $h$ is the depth between the measurement point and the standard altitude of the bottom of the blast hole, $R$ is the distance between the measurement point and point $o$, $R=\sqrt{l^{2}+\left(d-h_{1}\right)^{2}}, \delta_{i}$ is the incident angle of the underwater shock wave, and $\delta_{r}$ is the corresponding reflection angle.

The factors that influence underwater shock wave peak overpressure $\Delta p$ at a measurement point in underwater drilling blasting are primarily $c$, the detonation heat of the explosives $Q_{v}, d$, hole spacing, row spacing, borehole diameter, overdrilling depth, stem length, rock properties, $H$, $h, l, p_{0}$, and $\rho_{0}$. The hole spacings, row spacings, borehole diameters, overdrilling depths, stem lengths, and rock properties were basically the same for each blasting during the test, and, therefore, we mainly considered the influences of $c, Q_{v}$, $d, H, h$, and $l$ on underwater shock wave peak overpressure. According to the $\pi$ theorem, the peak overpressure $\Delta p$ can be expressed as

$$
\Delta p=f\left(c, Q_{v}, d, H, h, L, p_{0}, \rho_{0}\right) .
$$

In addition

$$
\begin{aligned}
p_{0} & =1.013 *\left(1+\frac{h}{k}\right) \\
d+2 * H-1-h & =h_{1} ;
\end{aligned}
$$

therefore,

$$
\Delta p=f_{1}\left(c, Q_{v}, d, H, h, L, \rho_{0}\right) \text {. }
$$

Selecting $c, Q_{v}$, and $\rho_{0}$ as the independent variables and using the $\pi$ theorem, we can obtain the following dimensionless constants:

$$
\begin{aligned}
& \pi_{1}=\frac{\Delta p}{Q_{v} * \rho_{0}} \\
& \pi_{2}=\frac{d}{c^{1 / 3} \rho_{0}^{-1 / 3}} \\
& \pi_{3}=\frac{H}{c^{1 / 3} \rho_{0}^{-1 / 3}} \\
& \pi_{4}=\frac{h}{c^{1 / 3} \rho_{0}^{-1 / 3}} \\
& \pi_{5}=\frac{l}{c^{1 / 3} \rho_{0}^{-1 / 3}} .
\end{aligned}
$$

Therefore, the relationship between the underwater shock wave peak overpressure $\Delta p$ at a measurement point and the charge properties, blast hole mesh parameters, blasting environment and position of the measurement point can be expressed as

$$
\begin{gathered}
F\left(\frac{\Delta p}{Q_{v} * \rho_{0}}, \frac{d}{c^{1 / 3} \rho_{0}^{-1 / 3}}, \frac{H}{c^{1 / 3} \rho_{0}^{-1 / 3}}, \frac{h}{c^{1 / 3} \rho_{0}^{-1 / 3}},\right. \\
\left.\frac{l}{c^{1 / 3} \rho_{0}^{-1 / 3}}\right)=0,
\end{gathered}
$$

where $Q_{v}$ and $\rho_{0}$ are constant for any measurement point; therefore, (10) can be rewritten as

$$
\Delta p=F_{1}\left(\frac{d}{c^{1 / 3}}, \frac{H}{c^{1 / 3}}, \frac{h}{c^{1 / 3}}, \frac{l}{c^{1 / 3}}\right) .
$$

Define $\bar{d}=d / c^{1 / 3}, \bar{H}=H / c^{1 / 3}, \bar{h}=h / c^{1 / 3}$, and $\bar{l}=l / c^{1 / 3}$ as the proportional water depth in the blasting area, proportional distance between the center of the charge and the upper end surface of the blast hole, proportional depth between the measurement point and standard altitude of the bottom of the blast hole, and proportional horizontal distance between the measurement point and the explosive source. Equation (11) can be modified as follows:

$$
\Delta p=F_{1}\left(\frac{d}{c^{1 / 3}}, \frac{H}{c^{1 / 3}}, \frac{h}{c^{1 / 3}}, \frac{L}{c^{1 / 3}}\right)=a \bar{d}^{b_{1}} \bar{H}^{b_{2}} \bar{h}^{b_{3}} \bar{L}^{b_{4}},
$$

where $a, b_{1}, b_{2}, b_{3}$, and $b_{4}$ are undetermined coefficients. 
Taking the logarithms of both sides of (12) to transform the nonlinear equation into a linear equation yields

$$
\ln \Delta p=\ln a+b_{1} \ln \bar{d}+b_{2} \ln \bar{H}+b_{3} \ln \bar{h}+b_{4} \ln \bar{L} .
$$

Let $\ln \bar{d}=x_{1}, \ln \bar{H}=x_{2}, \ln \bar{h}=x_{3}, \ln \bar{L}=x_{4}, \ln \Delta p=x_{5}=y$, and $\ln a=b_{0}$; (13) then becomes

$$
y=b_{0}+b_{1} x_{1}+b_{2} x_{2}+b_{3} x_{3}+b_{4} x_{4}
$$

After analyzing the test results listed in Table 2 using the method of multiple linear regression, we can obtain that $b_{0}=$ $3.2435, b_{1}=0.4909, b_{2}=-0.0495, b_{3}=-1.4359$, and $b_{4}=$ -1.2312 . For the regression model, the complex correlation coefficient $R^{2}=0.9597$ and the adjustable decision coefficient $\bar{R}^{2}=0.9579$, both of which were very close to 1 , and the model estimate standard error $S_{y}=0.0829$, which was very close to 0 . Therefore, we can consider the imitative result better. Then,

$$
\begin{aligned}
\hat{y}= & 3.2435+0.4909 x_{1}-0.0495 x_{2}-1.4359 x_{3} \\
& -1.2312 x_{4} .
\end{aligned}
$$

By replacing $\hat{y}, x_{1}, x_{2}, x_{3}$, and $x_{4}$ with $\ln \Delta p, \ln \bar{H}$, $\ln \bar{h}, \ln \bar{d}$, and $\ln \bar{l}$, respectively, in (12), we can obtain the following revised formula for the underwater shock wave peak overpressure:

$$
\begin{aligned}
\Delta p= & 25.6232 * \bar{d}^{0.4909} * \bar{H}^{-0.0495} * \bar{h}^{-1.4359} \\
* \bar{l}^{-1.2312}(\mathrm{MPa}) &
\end{aligned}
$$

The ranges of application of the parameters are $0.6663 \leq \bar{d} \leq$ 2.8554, $0.3413 \leq \bar{H} \leq 0.9336,1.243 \leq \bar{h} \leq 3.3469$, and $6.9993 \leq \bar{l} \leq 14.8448$.

The values of the underwater shock wave peak overpressures were calculated using (16), and the calculated values and measured data were compared to obtain the relative error between them. Finally, we found that the relative errors were between $0.3 \%$ and $11.1 \%$, and there were only three measurement points with relative errors exceeding $10 \%$. Therefore, we can hold that the revised underwater shock wave peak overpressure formula can describe the attenuation characteristics of the underwater shock waves better than the traditional exponential attenuation formula.

\section{The Significance Order of Influence and Influence Mechanism}

In the regression model, the regression variables $x_{1}, x_{2}, x_{3}$, and $x_{4}$ were sorted according to the significance order of influence on the underwater shock wave peak overpressure expected value $\hat{y}$ determined using a $t$-test. Table 3 shows the test statistics $t_{i}$.

According to the results shown in Table 3, the order of the significances of the influences of the regression variables on the underwater shock wave peak overpressure expected value $\widehat{y}$ from high to low was $x_{3}, x_{4}, x_{1}$, and $x_{2}$; that is, the effect of
TABLE 3: Test statistics $t_{i}$.

\begin{tabular}{ccccc} 
& $x_{1}$ & $x_{2}$ & $x_{3}$ & $x_{4}$ \\
\hline$t_{i}$ & 1.9726 & -0.1991 & -5.7704 & -4.9477 \\
\hline
\end{tabular}

$h$ on the underwater shock wave peak overpressure was the most significant and was followed by those of $l$ and $d$, and the effect of $H$ on the underwater shock wave peak overpressure was relatively the weakest.

As shown in Figure 3, the underwater shock wave at a measurement point mainly consists of a shock wave produced by detonation products as they directly rush out into the water from the blast hole. For underwater drilling blasting, the detonation products that rush out of the blast hole are produced primarily by the charge located in the upper part of the blast hole. In actual construction processes, all blast holes are basically free of stemming, the detonation products therefore can rush out of the blast hole without being blocked, and the length of the charge whose detonation products rush out of the blast hole basically remains unchanged. Therefore, when other influence factors remain the same, the change in $H$ that indicates the position of the charge in the blast hole has the relatively the weakest effect on underwater shock wave peak overpressure. When $H$ becomes larger, the distance from the charge to the upper end surface of the blast hole increases, which means that there are more restrictions on the expansion of the detonation products, and the generated shock wave becomes relatively smaller.

After detonation, because the charge weight per delayed interval was large, the blast hole was almost free of stemming, and the detonation products directly rushed out of the blast hole and formed an obvious water column above the areas of the explosion. When the water depth $d$ in the explosion area increases and the remaining blasting parameters remain constant, fewer detonation products rush out of the water surface, and the residual energy from the explosion in the water increases. At the same time, as $d$ becomes larger, the attenuation of the shock wave decreases. Hence, as $d$ increases, the underwater shock wave peak overpressure at a measurement point increases, and $d$ has a certain extent of effect on the underwater shock wave peak overpressure.

As shown in Figure 5, $R=\sqrt{l^{2}+\left(d-h_{1}\right)^{2}}=$ $\sqrt{l^{2}+(h+1-2 * H)^{2}}$. The differences between $l$ and $R$ were between $0.0917 \mathrm{~m}$ and $1.5043 \mathrm{~m}$, and therefore the propagation distance $R$ of the underwater shock wave was primarily determined by $l$. As the propagation distance increased, the wave front of underwater shock wave became large, and the per unit area energy of the wave front became small, which accompanied the energy loss. Therefore, $l$ had a large influence on underwater shock wave peak overpressure, and with the increase in $l$, the underwater shock wave peak overpressures became small.

As shown in Figure 5, when the shock wave arrived at the free water surface along " $k$, " it was reflected as a sparse wave that could be deemed as having been generated by the mirror virtual wave source $o^{\prime}$ and propagating along " $k U$." When reaching the measurement point $U$, the reflected sparse wave 
TABLE 4: Test statistics $t_{i}$.

\begin{tabular}{ccccc} 
& $x_{1}$ & $x_{2}$ & $x_{3}$ & $x_{4}$ \\
\hline$t_{i}$ & 1.9626 & -0.2643 & -5.5724 & -5.024 \\
\hline
\end{tabular}

was superimposed on the incident shock wave. The incident angle $\delta_{i}=\sin ^{-1}\left(d / l_{1}\right)$, where $l_{1}=d \times \sqrt{\left(d+h_{1}\right)^{2}+l^{2}} /\left(d+h_{1}\right)$. According to the literature, when $\delta_{i}<\delta^{*}$, the sparse wave will catch up and surpass the incident shock wave, causing an irregular reflection.

$$
\delta^{*}=\sqrt{\frac{(n+1) * p_{m}}{(2 * n * B)}},
$$

where $n=7.15, B=304.5 \mathrm{MPa}$, and $p_{m}$ is the peak overpressure of the incident shock wave at point $k$ [14].

According to the above analysis of the influence of the free water surface on the shock wave, the calculated results of the incident angles of the shock waves and the critical angles of irregular reflection under various test conditions are shown in Table 2. As seen in Table 2, the incident angles were between $0.077416 \mathrm{rad}$ and $0.264859 \mathrm{rad}$, and the critical angles of the irregular reflection were between $0.025717 \mathrm{rad}$ and $0.067419 \mathrm{rad}$; therefore, all the measurement points satisfied $\delta_{1}>\delta^{*}$, which indicated that the reflections of all the incident shock waves on the free water surface were regular reflections and that the reflected sparse waves had no effect on the peak overpressures of the incident shock waves.

When other influence factors remained constant, the propagation distance of underwater shock wave changed with changes in $h$. Because the attenuation of a shock wave is related to propagation distance, $l$ was therefore replaced with $R$ in the revised formula for the underwater shock wave peak overpressure to eliminate the effect of $h$ on the underwater shock wave peak overpressure. After reanalysis, we obtained $b_{0}=3.2702, b_{1}=0.4909, b_{2}=-0.0661, b_{3}=-1.3937, b_{4}=$ -1.2526 , complex correlation coefficient $R^{2}=0.9598$, the adjustable judgment coefficient $\bar{R}^{2}=0.958$, and the estimated standard error $S_{y}=0.082$. The regression variables $x_{1}, x_{2}, x_{3}$, and $x_{4}$ were sorted according to the order of the significances of their influences on the underwater shock wave peak overpressure expected value $\hat{y}$; that is, a $t$-test was performed. Table 4 presents the test statistics $t_{i}$. From the data presented in the table, we can see that the influence of each factor on the underwater shock wave peak overpressure obeyed the same order of significance compared with the model in which $l$ was not replaced with $R$. Therefore, we can consider that the influence of the change of $h$ on $R$ cannot explain the influence law of $h$ on the underwater shock wave peak overpressure.

Table 2 and Figure 6 show that, for measurement points, when $d, H, l$, and $c$ were the same, the incident angle of the shock wave increased with decreasing $h$, and underwater shock wave peak overpressure increased with increasing incident angle. According to the analysis of the curve of the typical pressure time history of the underwater shock wave, to some extent, the maximum value of underwater shock wave

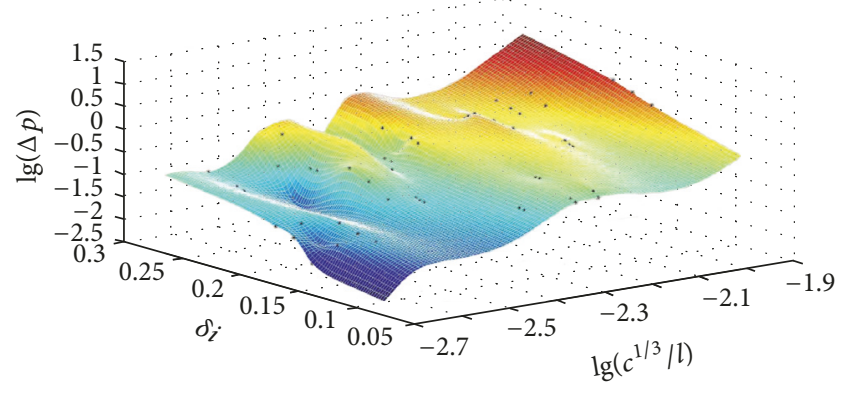

Figure 6: Mountain surface and scatter figure of the shock wave peak overpressure.

peak overpressure was dependent on the superposition of the shock waves generated by the blast holes with the same delay times. When variables $d, H, l$, and $c$ were the same, the intensities of the shock waves generated by each single blast hole were basically equal for all measurement points, but for the measurement points with larger angles of incidence, the shock wave arrived early and was less subjected to attenuation from the sparse wave that arose from the free water surface regular reflection. Therefore, when superimposed with shock waves arriving later, larger synthetic peak pressures can be produced. To summarize, $h$ had the most significant effect on the underwater shock wave peak overpressure, and the underwater shock wave peak overpressure increased with decreasing $h$.

\section{Conclusions}

Based on an analysis of measured underwater shock waves derived from underwater drilling blasting under complex sea conditions, this research presented the following conclusions.

(1) Compared with a general underwater shock wave, an underwater shock wave from underwater drilling blasting demonstrates many different characteristics, including successive multiple peak values, and the maximum value of peak overpressure depends to some extent on the superposition of shock waves generated by each single blast hole.

(2) Using dimensional analysis, we deduced a revised formula for underwater shock wave peak overpressure. By analyzing the relative error between the measured and predicted values, we found that the revised formula can better describe the propagation characteristics of underwater shock waves.

(3) The significance of the influence of each factor on underwater shock wave peak overpressure was, in order, $h$, $l, H$, and $d$.

(4) Unlike general underwater blasting, the maximum values of underwater shock wave peak overpressures arising from underwater drilling blasting depend to some extent on superimpositions of shock waves generated by each blast hole which have the same delay times. Therefore, within the regular reflection range, peak overpressure increases with increasing incident angle (i.e., decreasing $h$ ). 


\section{Conflicts of Interest}

The authors declare that they have no conflicts of interest.

\section{Acknowledgments}

This work was supported by grants from the Department of Infrastructure Barracks and National Science-Technology Support Plan (Grants nos. BY209J033 and 2012BAK05B01). Their contributions to bringing this project to fruition are much appreciated.

\section{References}

[1] X. Y. Song et al., Comprehensive Safety engineering research institute Edit, Japanese. Underwater Blasting, Central South University of Technology Press, Chang Sha, China, 1989.

[2] P. D. Jepson, M. Arbelo, R. Deaville et al., "Gas-bubble lesions in stranded cetaceans," Nature, vol. 425, no. 6958, pp. 575-576, 2003.

[3] W.-J. Jung, Y. Ogata, S. Kubota, H. Shimada, and K. Matsui, "Relationship of fragmentation of cylindrical rock specimen and incident underwater shock wave," Kayaku Gakkaishi/Journal of the Japan Explosives Society, vol. 63, no. 6, pp. 404-409, 2002.

[4] X. Su, Shockwave Monitoring and Transmission Characteristics Analysis of Underwater Explosion at Xiamen Harbor (A), Xiamen University, Xiamen, China, 2007.

[5] S. L. Luo, X. Sh. Ye, W. B. Gu, W. H. Liu, T. H. Yang, and P. X. Zhang, "The status of underwater explosion research," Engineering Blasting, vol. 5, no. 1, pp. 84-87, 1999.

[6] Y. X. Peng, L. Wu, Y. Su, and C. H. Chen, "Study on the effect of elevation on the prediction of underwater drill and blasting vibration frequency," Geosystem Engineering, vol. 19, no. 4, pp. 170-176, 2016.

[7] W. B. Gu, J. H. Chen, Zh. X. Wang, Zh. H. Wang, J. Q. Liu, and M. Lu, "Experimental Study on the Measurement of Water Bottom Vibration Induced by Underwater Drilling Blasting," Shock and Vibration, vol. 2015, Article ID 496120, 8 pages, 2015.

[8] G. R. Tripathy and R. R. Shirke, "Underwater drilling and blasting for hard rock dredging in indian ports - a case study," ICWRCOE Aquatic Procedia, vol. 4, pp. 248-255, 2015.

[9] Y. Zh. Sun, Y. Long, L. Zh. Shao, and X. B. Xie, "Experimental investigation of shock wave in water of underwater drilling blasting," Engineering Blasting, vol. 13, no. 4, pp. 15-19, 2007.

[10] Q. Guo, Parameters' Optimization Analysis in Underwater Blasting, Wuhan University of Technology, Wuhan, China, 2005.

[11] H. Wang, Numerical Simulation of Underwater Drilling Blasting, North University of China, Taiyuan, China, 2014.

[12] X. W. Chai, Propagation Characteristics of Water Shock in Underwater Borehole Blasting, Wuhan University of Technology, Wuhan, China, 2009.

[13] X. Q. Liang, Underwater Explosion Technology, Chemical industry Press, Beijing, China, 2013.

[14] R. H. Cole, Underwater Explosions, Dover Publications, New York, NY, USA, 1965. 


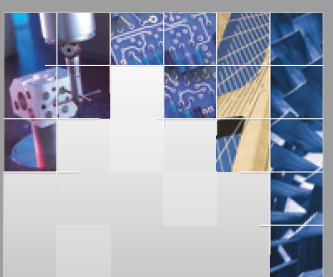

\section{Enfincering}
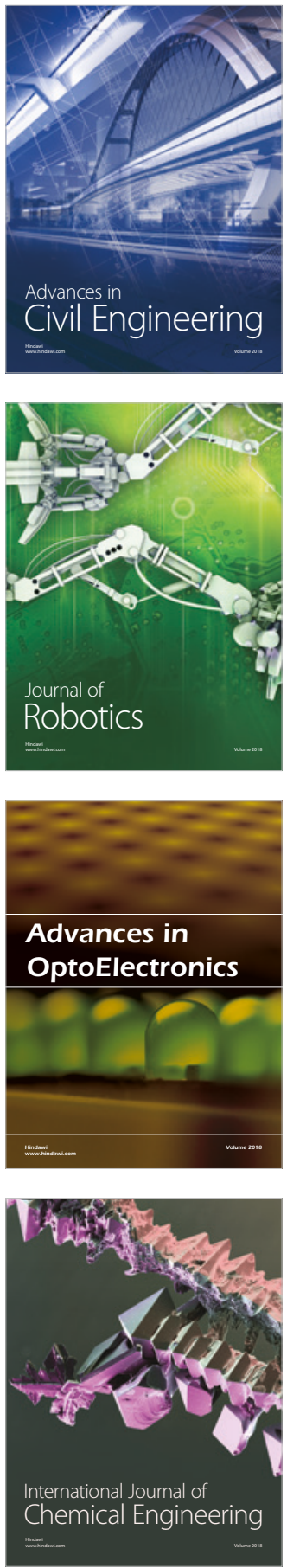

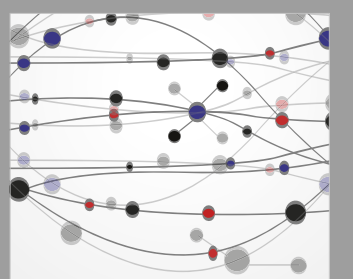

\section{Rotating \\ Machinery}

The Scientific World Journal

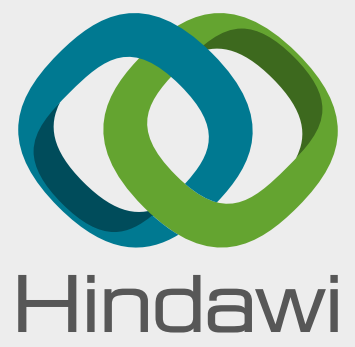

Submit your manuscripts at

www.hindawi.com
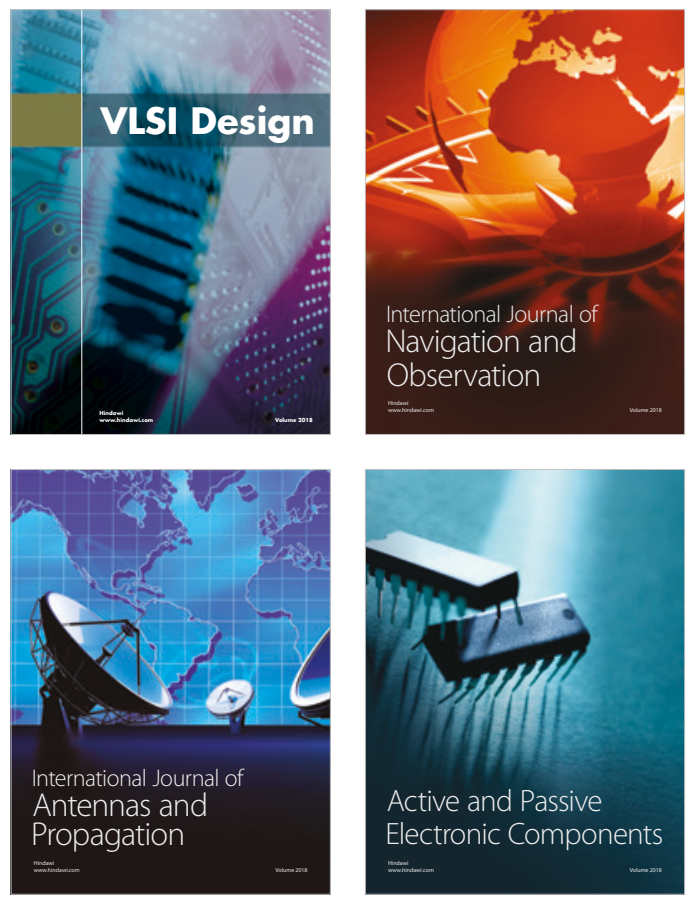
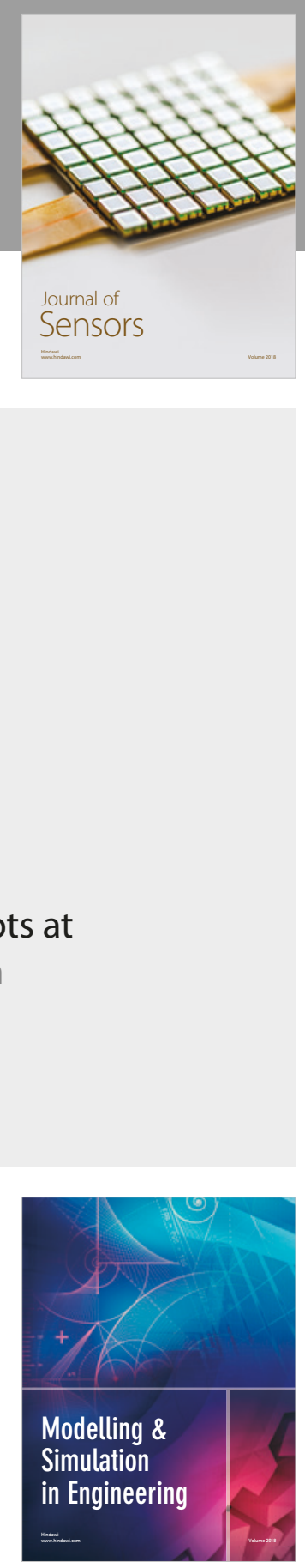

\section{Advances \\ Multimedia}
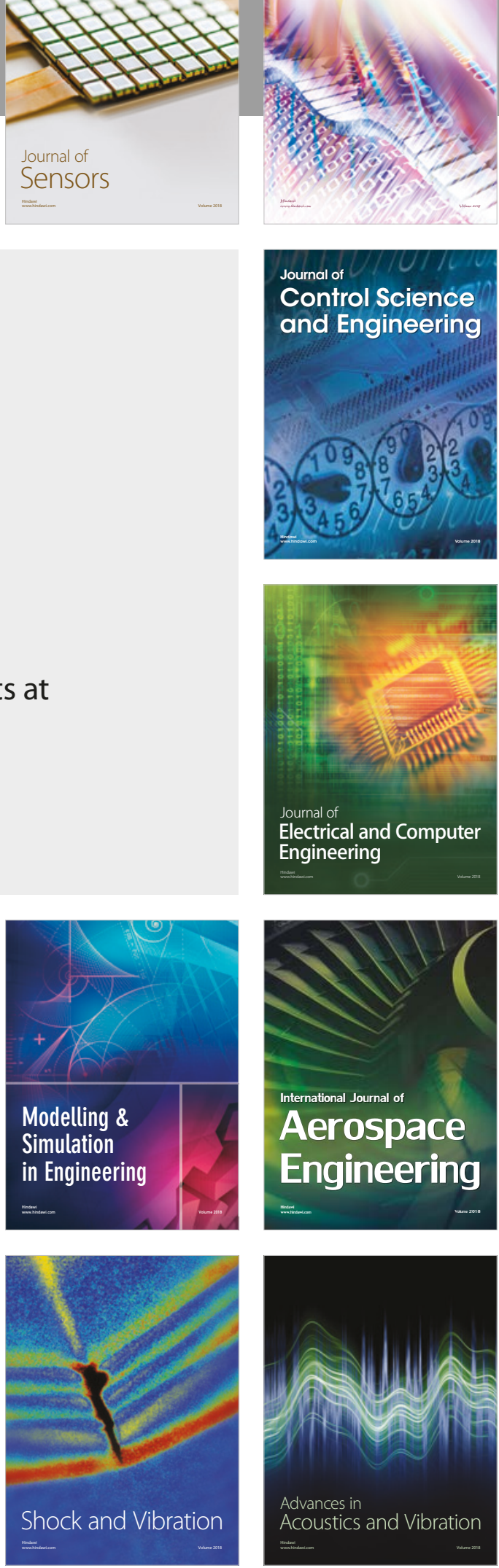